and LVEF was measured. Serum creatinine (Scr) was measured before and after administered the medication 24 h, 48 h, 72 h, 7 days and 14 days using simplified MDRD equation to calculate estimated glomerular filtration rate (eGFR). Recording the major adverse cardiac events (MACE) occurrence within $30 \mathrm{~d}$.

Results rhBNP group has a less dyspnoea time than the control group; The plasma BNP levels significantly lower than before treatment at different time point in the two groups. The LVEF was significantly higher in treatment group compared with baseline levels after treatment $24 \mathrm{~h}$, while LVEDD significantly decreased even after discontinuation the treatments, which remain so when the 30 days. The LVEF and LVEDD improvements in rhBNP group were significantly better than in the control group after treatment 24 h, 14 days; At day 7 after PCI, the SCr had lowered to the baseline level in the rhBNP group. The eGFR after PCI was higher in the rhBNP group than that in the control group. The occurrence of CIN was signicantly lower in the rhBNP group than in the control group. The MACE event of 30 days in rhBNP group was significantly lower than the control group.

Conclusion rhBNP can promptly and effectively improve the heart function, reduce the incidence of MACE rate in acute myocardial infarction with heart failure patients, which also had a renal function protective effect in patients with and decreased incidence on CIN.

\section{e0628 CLINICAL ANALYSIS OF ACUTE MYOCARDIAL INFARCTION IN YOUNG PATIENTS}

doi:10.1136/hrt.2010.208967.628

Fu Xianghua, Wu Weili, Zhang Wenjing, Wang Xuechao, Wang Yanbo, Jiang Yunfa, Hao Guozhen. The Second Hospital of Hebei Medical University

Objective To investigate the clinical characteristics of acute myocardial infarction (AMI) in young patients.

Methods We carried out the contrasting analysis in the clinical data between 45 young patients (age $\leq 45$ years old) and 52 old patients (age $\geq 60$ years old).

Results Young AMI patients were often male, and had the typical clinical manifestations. The smoking rate, hyperfibrinogenemia rate and positive family history rate of the young people group were markedly higher than those of the old people group $(p<0.05)$. The morbidity rate of patients with single coronary artery atherosclerosis was high in the young people group. The morbidity rate of patients with multiple coronary artery atherosclerosis was high in the old people group. The patients in the old people group who complicated with cardiac aneurysm, arrhythmia, heart failure, cardiac shock were much more than those in the young people group $(p<0.05)$.

Conclusion Smoking, hyperfibrinogenemia and positive family history are main causes of AMI in young patients. Young AMI patients had the typical clinical manifestations with simple coronary lesion. The complications in the young people group are less than those in the old people group, and the prognosis was better than old cases.

\section{e0629 THE EFFECTS OF SLEEP APNOEA SYNDROME ON MYOCARDIAL ISCHAEMIA IN PATIENTS WITH CORONARY HEART DISEASE DURING NIGHT}

doi:10.1136/hrt.2010.208967.629

Fu Xianghua, Pang Jiangna, Wang Xuechao, Wang Yanbo, Jiang Yunfa, Wu Weili, Hao Guozhen, Gu Xinshun. The Second Hospital of Hebei Medical University

Objective 1. To investigate the relationship between sleep apnoea syndrome (SAS) and myocadial ischaemic events in patients with coronary heart disease (CHD). 2. To compare the differences of age, gender distribution, coronary angiography results in CHD patients with and without SAS. 3. To compare the differences of C-reactive protein (CRP) and haemoglobin levels in CHD patients with and without SAS.

Methods $25 \mathrm{CHD}$ patients with typical symptoms of angina and ECG changes were enrolled in this study. After overnight polysomnography (PSG), all the cases were monitored by portable device at night for 7 days in order to exclude the conditions that the cases did not sleep or had waked, apnoea and hypopnoea events were recorded during 24: 00-4:00. Blood samples were collected 5-10 min after monitoring, and the levels of haemoglobin and Creactive protein were examined.

Results 1 . The incidence of myocardial ischaemia caused by apnoea and low ventilation was significantly higher in CHD patients with SAS. 2. There were significant differences between the two groups in the decrease of oxygen desaturation and the increase of heart rate. 3 . BMI in CHD patients was significantly higher in those with SAS. There were more multi-vessel lesions and long lesions in CHD patients with SAS $(p<0.05)$. The level of haemoglobin and C-reactive protein were much higher in CHD patients with SAS.

Conclusion 1. The incidence of SAS is much higher in patients with $\mathrm{CHD}$, and the incidence of myocardial ischaemic events is higher in $\mathrm{CHD}$ patients with SAS. and the more seriuos respiratory disorders, the more easily myocardial ischaemia happens. With apnoea related to myocardial ischaemia and oxygen reduction, has nothing to do with the heart rate. 2. Lesions of SAS in patients with coronary heart disease are heavier than Simple CHD group in coronary angiography. BMI of SAS in patients with coronary heart disease are high than Simple CHD group. 3. The levels of CRP and haemoglobin are higher in CHD patients with SAS.

\section{e0630 THE INFLUENCE ON THE PLATELET FUNCTION OF DIFFERENT STATINS COMBINED WITH LOADING DOSE CLOPIDOGREL IN PATIENTS WITH ACUTE CORONARY SYNDROME}

doi:10.1136/hrt.2010.208967.630

Fu Xianghua, Wang Oian, Wang Yanbo, Wang Xuechao, Hao Guozhen, Jiang Yunfa, Fan Weize, Gu Xinshun. The Second Hospital of Hebei Medical University

Objective To investigate clinical effects of clopidogrel combined with simvastatin or fluvastatin on the platelet aggregation rate (PAR), platelet activation marker CD62P and the incidence of major adverse cardiovascular events (MACE) in patients with ACS.

Methods From April 2008 to December 2009, one hundred patients (79 male and 21 female, average age $61.46 \pm 12.84$ years) who had been diagnosed as ACS were enrolled into this study. These cases were randomly divided into two groups, the Group $A$ ( $n=50$, treated with simvastatin $20 \mathrm{mg}$ per night); the Group $B$ ( $n=50$, treated with fluvastatin $40 \mathrm{mg}$ per night). Detailed clinical information was collected. PAR, CD62P, alanine aminotransferase (ALT), and aspartate aminotransferase (AST) of the two groups were measured. All cases received clopidogrel (a loading dose of $300 \mathrm{mg}$ and then $75 \mathrm{mg}$ daily), aspirin and Low molecular weight heparin. The MACE within 14 days were recorded.

Results There was no significant differences in baseline between the Group A and Group B. There was no significant differences in the PAR and expression rate of CD62P after $300 \mathrm{mg}$ clopidogrel ( $p>0.05) .1 \mathrm{~h}$ after treated with statins the expression rate of CD62P and PAR in the two groups were lower than that before treated with statins $(p<0.05)$. After $14 d$ treated with statins the expression rate of CD62P and PAR were still lower than that before treated with statins $(p<0.05)$. There were no significant increase of ALT and AST in the both groups $(p>0.05)$. After the above-mentioned medical treatment, the expression rate of CD62P and PAR in the two groups 